\title{
The power of immunotherapy plus platinum-based chemotherapy for locally advanced or early stage non-small cell lung cancer
}

\author{
Paul Hofman ${ }^{1,2,3}$ \\ ${ }^{1}$ Université Côte d'Azur, CHU Nice, FHU OncoAge, Laboratory of Clinical and Experimental Pathology, Pasteur Hospital, Nice, France; \\ ${ }^{2}$ Université Côte d'Azur, CNRS, INSERM, IRCAN, FHU OncoAge, Team 4, Nice, France; ${ }^{3}$ Université Côte d'Azur, CHU Nice, FHU OncoAge, \\ Hospital-Integrated Biobank (BB-0033-00025), Nice, France \\ Correspondence to: Paul Hofman, MD, PhD. Laboratory of Clinical and Experimental Pathology, Pasteur Hospital, 30 avenue de la voie Romaine, \\ BP69, 06001 Nice cedex 01, France. Email: hofman.p@chu-nice.fr. \\ Provenance and Peer Review: This article was commissioned by the Editorial Office, Annals of Translational Medicine. The article did not undergo \\ external peer review. \\ Comment on: Hu H, Zou S, Xu R, et al. Conversion therapy from N3 unresectable lung adenocarcinoma to radical surgery: a case report. Ann Transl \\ Med 2019;7:590.
}

Submitted Dec 28, 2019. Accepted for publication Jan 16, 2020.

doi: 10.21037/atm.2020.01.91

View this article at: http://dx.doi.org/10.21037/atm.2020.01.91

Immunotherapy alone or combined with chemotherapy has drastically reconfigured first-line treatment of patients with non-small cell lung carcinoma (NSCLC) $(1,2)$. Until recently, treatment concerned stage IIIB/IV tumors showing no EGFR, ALK, ROS1, BRAF or NTRK genomic alterations (1).

The treatment of locally advanced initially non surgically resectable tumors [stage IIIA (T1-T4, N0-N2)] is complex and depends on mediastinal lymph node staging. The goal of the different therapeutic strategies, intended as a permanent cancer cure, was to provide patients with thoracic surgery. So, until recently the gold standard treatment of these tumors was platinum-based chemotherapy or radio-chemotherapy prior to surgery, sometimes in association with adjuvant radiotherapy or radio-chemotherapy in case of a residual tumor. For stage IIIB (N3) non resectable tumors, radiochemotherapy, chemotherapy or immunotherapy alone or combined immunotherapy and chemotherapy are proposed for first-line treatment of NSCLC wild-type for the genes cited above.

Following the positive result of the PACIFIC clinical trial, patients with locally advanced non resectable (stage IIIA) NSCLC wild-type for EGFR and $A L K$ can be treated with radio-chemotherapy prior to immunotherapy consolidation (durvalumab) (3). This sequential therapy followed by surgical resection of the tumor has been recently approved by the FDA and EMA $(4,5)$. This new therapeutic strategy demonstrated improved overall survival in comparison of that obtained with chemotherapy or radio-chemotherapy prior to surgery, irrespective of the PD-L1 status (4,5). Different treatments which associate other immunotherapy molecules (notably nivolumab or atezolizumab) and chemotherapy are currently proposed in clinical trials for stage III NSCLC $(6,7)$. More recently, clinical trials using neoadjuvant immunotherapy are ongoing for early stage (stages I-II) NSCLC (8-11). Finally, other clinical trials associating adjuvant immunotherapy alone or in combination with adjuvant chemotherapy or adjuvant radio-chemotherapy are ongoing $(7,11)$.

A recent publication by $\mathrm{Hu}$ and colleagues concerned a patient with stage IIIB (T1bN3M0) EGFR and ALK wildtype lung adenocarcinoma (12). This patient was treated with pembrolizumab and chemotherapy (association of pemetrexed and carboplatin). Complete clinical regression of the metastatic lymph nodes was observed after 4 cycles of treatment giving a cT1bN0M0 tumor (12). Since the disease was stable after these 4 cycles, surgery for complete tumor resection was indicated (12). Postoperative histological analysis showed no residual tumor cells in the 39 resected lymph nodes and a few PD-L1 negative tumor cells associated with massive lymphocytic infiltrates in the primary tumor (12). This attested to a high level of response to the neoadjuvant treatment. Moreover, due to the result of the histological analysis and the absence of some driver mutations, no adjuvant therapy 
was administered. No recurrence of the disease was noted at the time of the publication 33 months after surgery (12). Nonetheless, this dramatic result concerned: (I) a smallsized tumor (T1b; $>1 \mathrm{~cm}$ and $<2 \mathrm{~cm}$ ) corresponding to an $E G F R / A L K$ wild-type adenocarcinoma (12); (II) a tumor with $90 \%$ PD-L1 positive tumor cells; (III) a tumor with a high tumor mutational burden (TMB) (11 muts/Mb) (12). Thus, this small tumor possessed two positive predictive biomarkers for immunotherapy responsiveness. In the absence of residual tumor cells in the lymph nodes and in the presence of a low percentage of residual tumor cells in the completely resected primary tumor, the decision to not provide adjuvant treatment (immunotherapy and/or chemotherapy) was justified (12). No targeted therapy was justified after surgery since this tumor was $E G F R$ and $A L K$ wild-type, even if the ROS1, BRAF, NTRK, MET, RET and HER 2 status was not provided (12).

So, combined neoadjuvant immunotherapy and chemotherapy prior to surgery can be proposed for stage IIIB NSCLC in the absence of any drugable genomic alteration and may lead to complete surgical tumor resection. However, we need to keep in mind that this is a unique case report and that future clinical trials including a larger number of patients are mandatory to confirm the possible benefit of this therapeutic strategy for stage IIIB NSCLC. In this context, different biomarkers should be examined and assessed before proposing this treatment. We may wonder if this therapeutic strategy can be provided only in the case of EGFR, ALK, ROS1, BRAF and NTRK wildtype NSCLC with more than $50 \%$ of tumor cells expressing PD-L1 and with a high TMB. Moreover, the histological results obtained after surgery could suggest or not providing adjuvant therapy, notably adjuvant immunotherapy. In this regard the assessment of the resected specimen needs to be complete and to integrate the different morphological parameters recently described $(13,14)$. One of the main histological criteria to assess is the percentage of residual tumor cells since major response is defined by the presence of less than $10 \%$ tumor cells $(13,14)$. However, other biological parameters could allow better prediction of the histological response to neoadjuvant immunotherapy such as the assessment of a higher intra tumoral and blood $\mathrm{T}$ cell receptor clonality (15). Besides the histological and the biological parameters, the delay in surgical resection of stage III NSCLC after neoadjuvant immunotherapy needs to be controlled and certainly standardized since this delay can have an impact on overall survival (16). The delay needs to be further established according to the different neoadjuvant strategies, notably for stages IIIA or IIIB NSCLC.

The development of neoadjuvant immunotherapies requires integrating and combining in the near future several predictive biomarkers of treatment responsiveness, as well as some biomarkers of resistance and of therapeutic toxicity. In this context, PD-L1 immunohistochemistry and the TMB have their limits (17-20). There is an urgent need to clinically validate some other tissue biomarkers but also some new blood biomarkers to better adapt neoadjuvant treatments (21). Thus, to be able to predict effective neoadjuvant immunotherapy leading to a complete surgical resection in NSCLC patients with not only stage I-IIIA but also stage IIIB tumors may be possible.

\section{Acknowledgments}

The author wishes to thank the Ligue Départementale 06, the Conseil Départemental 06 des Alpes Maritimes, and the Cancéropôle PACA for their support.

Funding: None.

\section{Footnote}

Conflicts of Interest: $\mathrm{PH}$ serves as an unpaid editorial board member of Annals of Translational Medicine from Jul 2018 to Jun 2020.

Ethical Statement: The author is accountable for all aspects of the work in ensuring that questions related to the accuracy or integrity of any part of the work are appropriately investigated and resolved.

Open Access Statement: This is an Open Access article distributed in accordance with the Creative Commons Attribution-NonCommercial-NoDerivs 4.0 International License (CC BY-NC-ND 4.0), which permits the noncommercial replication and distribution of the article with the strict proviso that no changes or edits are made and the original work is properly cited (including links to both the formal publication through the relevant DOI and the license). See: https://creativecommons.org/licenses/by-nc-nd/4.0/.

\section{References}

1. Dafni U, Tsourti Z, Vervita K, et al. Immune checkpoint inhibitors, alone or in combination with chemotherapy, as first-line treatment for advanced non-small cell lung 
cancer. A systematic review and network meta-analysis. Lung Cancer 2019;134:127-40.

2. Peters S, Reck M, Smit EF, et al. How to Make the Best Use of Immunotherapy as First-Line Treatment for Advanced/Metastatic Non-Small-Cell Lung Cancer. Ann Oncol 2019. doi: 10.1093/annonc/mdz109.

3. Antonia SJ, Villegas A, Daniel D, et al. Durvalumab after Chemoradiotherapy in Stage III Non-Small-Cell Lung Cancer. N Engl J Med 2017;377:1919-29.

4. FDA expands approval of Imfinzi to reduce the risk of non-small cell lung cancer progressing. Available online: https://www.fda.gov/NewsEvents/Newsroom/ PressAnnouncements/ucm597217.htm

5. Peters S, Dafni U, Boyer M, et al. Position of a panel of international lung cancer experts on the approval decision for use of durvalumab in stage III non-smallcell lung cancer (NSCLC) by the Committee for Medicinal Products for Human Use (CHMP). Ann Oncol 2019;30:161-5

6. Lin SH, Lin Y, Price J, et al. DETERRED: PD-L1 blockade to evaluate the safety of lung cancer therapy using carboplatin, paclitaxel, and radiation combined with MPDL3280A (atezolizumab). J Clin Oncol 2017;35:3064.

7. Yeh J, Marrone KA, Forde PM. Neoadjuvant and consolidation immuno-oncology therapy in stage III nonsmall cell lung cancer. J Thorac Dis 2018;10:S451-9.

8. Forde PM, Chaft JE, Smith KN, et al. Neoadjuvant PD-1 Blockade in Resectable Lung Cancer. N Engl J Med 2018;378:1976-86.

9. Forde PM, Chaft JE, Pardoll DM. Neoadjuvant PD-1 Blockade in Resectable Lung Cancer. N Engl J Med 2018;379:e14.

10. Rosner S, Reuss JE, Forde PM. PD-1 Blockade in EarlyStage Lung Cancer. Annu Rev Med 2019;70:425-35.

11. Sandler JE, D'Aiello A, Halmos B. Changes in store for early-stage non-small cell lung cancer. J Thorac Dis 2019;11:2117-25.

12. $\mathrm{Hu} \mathrm{H}, \mathrm{Zou} \mathrm{S}, \mathrm{Xu}$ R, et al. Conversion therapy from N3 unresectable lung adenocarcinoma to radical surgery: a case report. Ann Transl Med 2019;7:590.

Cite this article as: Hofman P. The power of immunotherapy plus platinum-based chemotherapy for locally advanced or early stage non-small cell lung cancer. Ann Transl Med 2020;8(5):151. doi: 10.21037/atm.2020.01.91
13. Blumenthal GM, Bunn PA Jr, Chaft JE, et al. Current Status and Future Perspectives on Neoadjuvant Therapy in Lung Cancer. J Thorac Oncol 2018;13:1818-31.

14. Cottrell TR, Thompson ED, Forde PM, et al. Pathologic features of response to neoadjuvant anti-PD-1 in resected non-small-cell lung carcinoma: a proposal for quantitative immune-related pathologic response criteria (irPRC). Ann Oncol 2018;29:1853-60.

15. Zhang J, Ji Z, Caushi JX, et al. Compartmental analysis of $T$ cell clonal dynamics as a function of pathologic response to neoadjuvant PD-1 blockade in resectable non-small cell lung cancer. Clin Cancer Res 2019. [Epub ahead of print].

16. Rice JD, Heidel J, Trivedi JR, et al. Optimal Surgical Timing after Neoadjuvant Therapy for Stage IIIa NonSmall Cell Lung Cancer. Ann Thorac Surg 2020;109:842-7.

17. Heeke S, Hofman P. Tumor mutational burden assessment as a predictive biomarker for immunotherapy in lung cancer patients: getting ready for prime-time or not? Transl Lung Cancer Res 2018;7:631-8.

18. Ilie M, Long-Mira E, Bence C, et al. Comparative study of the PD-L1 status between surgically resected specimens and matched biopsies of NSCLC patients reveal major discordances: a potential issue for anti-PD-L1 therapeutic strategies. Ann Oncol 2016;27:147-53.

19. Kazdal D, Endris V, Allgäuer M, et al. Spatial and Temporal Heterogeneity of Panel-Based Tumor Mutational Burden in Pulmonary Adenocarcinoma: Separating Biology From Technical Artifacts. J Thorac Oncol 2019;14:1935-47.

20. Thunnissen E, Kerr KM, Dafni U, et al. Programmed death-ligand 1 expression influenced by tissue sample size. Scoring based on tissue microarrays' and cross-validation with resections, in patients with, stage I-III, non-small cell lung carcinoma of the European Thoracic Oncology Platform Lungscape cohort. Mod Pathol 2019. [Epub ahead of print].

21. Hofman P, Heeke S, Alix-Panabières C, et al. Liquid biopsy in the era of immuno-oncology: is it ready for prime-time use for cancer patients? Ann Oncol 2019;30:1448-59. 